

\title{
Liquid Phase Exfoliation of Antimonene: Systematic Optimization, Characterization and Electrocatalytic Properties
}

Received 00th January 20xx, Accepted 00th January 20xx DOI: $10.1039 / \times 0 \times x 00000 x$

Carlos Gibaja, ${ }^{a}$ Mhamed Assebban, ${ }^{\mathrm{b}, \mathrm{c}}$ Iñigo Torres, ${ }^{\mathrm{a}}$ Michael Fickert, ${ }^{\mathrm{c}}$ Roger Sanchis-Gual, ${ }^{\mathrm{b}}$ Isaac

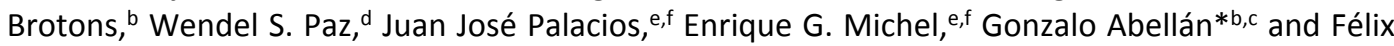
Zamora*a,f,g

\begin{abstract}
Antimonene, a novel group 15 two-dimensional material, is attracting great attention due to its outstanding physical and chemical properties. Despite its thermodynamic stability, the pronounced covalent character of the interlayer interactions imposes severe limitations on its exfoliation into mono- and few layers. Here, we developed a systematic study of the liquid phase exfoliation (LPE) with the aim to optimize the antimonene production in terms of concentration and dimensional anisotropy, investigating the most relevant experimental factors affecting the exfoliation: pre-processing of pristine antimony, solvent selection based on Hansen solubility parameters and ultrasound conditions. Moreover, an exhaustive characterization by means of Turbidimetry, XRD, Raman, XPS, AFM, SEM, XEDS and TEM has been carried out. Indeed, we reached concentration values of $c a .0 .368 \mathrm{~g} \cdot \mathrm{L}^{-1}$ ( yield of $37 \mathrm{wt} \%$ ), up to $\sim 30$ times higher compared to the topmost value so far reported, with ca. $50 \%$ of the nanolayers with heights between 2-10 nm, and lateral dimensions in the $40-300 \mathrm{~nm}$ range. Furthermore, it has been demonstrated that the yield of the process can be enhanced up to $~ 90 \mathrm{wt} \%$ by recycling the sediment to the process a maximum of 7 cycles. Moreover, we have illustrated the usefulness of this approach characterizing the electrochemical behaviour of antimonene as catalyst for the hydrogen evolution reaction (HER). This study provides important insights into the LPE and electrochemical properties of antimonene, allowing its large-scale production and paving
\end{abstract} the way for its application in fields of utmost importance such as energy storage and conversion or catalysis.

\section{Introduction}

Two-dimensional (2D) materials, such as graphene, ${ }^{1} \mathrm{~h}-\mathrm{BN}^{2}$ and $\mathrm{TMDs}^{3}$ have attracted great attention in materials science due to their outstanding properties and their application in electronic devices and energy storage, among others. ${ }^{4-7}$ However, for some specific applications it is necessary to find materials with a band-gap in the range of $0.1-1 \mathrm{eV}$. Black phosphorus (BP), has been the first candidate to show thickness-dependent band-gap values between $0.3-2 \mathrm{eV} .{ }^{8}$ Unfortunately, BP is particularly sensitive to the environmental

a. Departmento de Química Inorgánica and Institute for Advanced Research in Chemical Sciences (IAdChem), Universidad Autónoma de Madrid, 28049, Madrid, Spain.

b. Instituto de Ciencia Molecular (ICMol), Universidad de Valencia, Catedrático José Beltrán 2, 46980, Paterna, Valencia, Spain.

Department of Chemistry and Pharmacy \& Joint Institute of Advanced Materials and Processes (ZMP), Friedrich-Alexander-Universität Erlangen-Nürnberg (FAU), Dr.-Mack-Straße 81, 90762, Fürth, Germany.

d. Instituto de Física, Universidade Federal do Rio de Janeiro, Caixa Postal 68528, Rio de Janeiro, RJ 21941-972, Brazil.

e. Departamento de Física de la Materia Condensada Universidad Autónoma de Madrid, 28049 Madrid, Spain.

f. Condensed Matter Physics Center (IFIMAC) Universidad Autónoma de Madrid, 28049 Madrid, Spain.

g. Instituto Madrileño de Estudios Avanzados en Nanociencia (IMDEA-Nanociencia), Cantoblanco, Madrid E-28049, Spain

tElectronic supplementary information (ESI) available. See DOI: 10.1039/x0xx00000x.

‡ Carlos Gibaja and Mhamed Assebban contributed equally to this work. conditions and degrades in contact with water and oxygen both in the presence or absence of light. ${ }^{9-13}$ Here it comes the rise of alternative elemental group-15 layered materials, also called 2D-pnictogens, due to their reported environmental stability and their suitable band-gap for specific applications. ${ }^{14}$

Among these group-15 semiconductors, antimonene is the one that has been more intensely studied so far, due to its similarities with BP. ${ }^{15}$ Since it was initially predicted, ${ }^{16}$ and experimentally isolated, ${ }^{17,18}$ great efforts to find new applications for it, beyond (opto)-electronics, have been pushed up. ${ }^{19-25}$ For most of these applications, large quantities of antimonene nanolayers need to be produced in a scale-up way. ${ }^{18}$

Liquid phase exfoliation (LPE) has been extensively used to produce suspensions of many $2 \mathrm{D}$ materials mainly because is the most suitable method to produce single or few-layer ( $\mathrm{FL}$ ) materials in large-scale. ${ }^{26}$ LPE is a straightforward technique, appropriate for industrial scale-up and based on the solubility parameters theory. Ideally, the layered material is immersed into suitable solvent, i.e. the surface energy of the solvent and the layered material match with each other. The amount of energy necessary to overcome the van der Waals cohesive force between the layers of the crystal is usually, but not always, ${ }^{27}$ supplied in form of ultrasonic wave. Furthermore, LPE can also be applied to non-van-der-Waals solids such as iron ore hematite, ${ }^{28}$ being appropriate for those $2 \mathrm{D}$ materials exhibiting strong interlayer interactions with a greater covalent character 
such as heavy pnictogens ( $\mathrm{As}, \mathrm{Sb}$ and $\mathrm{Bi}$ ). These ultrasonic waves generate turbulence; shear forces and cavitation bubbles within the suspension. When the energy released is enough to overcome the cohesive energy of the material, exfoliation occurs generating a variety of mono- and few-layers, which are dispersed in the solvent. The stabilization of the so-formed suspension depends on the nanolayers interaction with the solvent since it may avoid particle aggregation. ${ }^{29,30}$ Therefore, LPE is also affected by the features of the crystal size of the starting material, solvent selection and the source of energy required to assist the exfoliation process.

We have reported for the first time in 2016 the isolation of $\mathrm{FL}$ antimonene nanolayers using LPE. ${ }^{18}$ Despite of the highquality of the FL antimonene nanolayers obtained in that work, we consider that developing a methodical study for enhancing the concentration of the suspensions $\left(0.00174 \mathrm{~g} \cdot \mathrm{L}^{-1}\right)$ can contribute to spread its potential applications. Herein, we report on a systematic analysis of the most significant parameters that control LPE process, in order to rationalize the production of $\mathrm{FL}$ antimonene suspensions. We have studied the effect in the LPE of antimony crystals of: $i$ ) the initial crystal size, ii) solvent used and iii) ultrasound parameters. In order to perform this fundamental study, we have designed a strategy based on three enchained steps. We have tried to optimize each step to use the best material before the next one, with the aim to produce suspensions with the higher $\mathrm{FL}$ antimonene concentration and the "dimensional anisotropy" (DA) ratio. We have defined DA parameter as the ratio between the length and height values of the nanolayers. DA provides a good estimation of the morphology of the nanolayers (the higher the DA ratio is, the thinner and larger the layers are). It is worth to remark here that in the case of antimonene, the short out-of-plane atom-toatom distances result in strong interlayer interactions and pose an additional limitation for the exfoliation.
The results obtained show that we can enhance up to ${ }_{1} g_{n} 30$

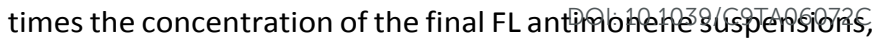
ca. $0.368 \mathrm{~g} \cdot \mathrm{L}^{-1}$ ( yield of $\left.37 \mathrm{wt} \%\right)$, compared to the highest concentration value so far reported, $c a .0 .014 \mathrm{~g} \cdot \mathrm{L}^{-1} .^{24}$ This has been achieved using a pre-processing step of the bulk antimony for reducing the crystallite thickness along the $c$-axis based on wet-ball milling in 2-butanol, followed by LPE step in an $\mathrm{NMP} / \mathrm{H}_{2} \mathrm{O}(4: 1)$ mixture assisted with a sonication tip. We have also observed that the DA ratio of the nanolayers becomes larger when using 2-butanol as a solvent (DA 27.6) keeping good concentration values (ca. $0.279 \mathrm{~g} \cdot \mathrm{L}^{-1}$ ). Besides, we also observed that the exfoliation yield can be enhanced up to $~ 90$ wt\% by recycling the sediment to the process a maximum of 7 cycles. Moreover, we found out different oxidation degrees depending on the solvent used. Last but not least, we have tested the electrochemical behaviour for the hydrogen evolution reaction (HER) on selected samples with disparate oxidation degrees and DA values showing significant differences. These results provide important knowledge about the exfoliation of antimonene and pave the way for the development of applications in fields of utmost importance such as energy storage and conversion or catalysis, to name a few.

\section{Experimental Section}

\section{Preparation of Ball-Milled Sb}

This procedure involves a pre-grinding process of the Sb crystals (Smart Elements, 99.9999\% purity) with an Agathe mortar giving rise to a so-called grinded $\mathrm{Sb}$. A $20 \mathrm{~mL}$ ball mill reactor (IKA Ultra-Turrax Tube Drive Control) was charged with $200 \mathrm{mg}$ of grinded $\mathrm{Sb}$ and 30 stainless steel balls, to complete a total volume of $7.5 \mathrm{~mL}$. Then, the mixture stirred in the reactor for 60 min. at $3000 \mathrm{rpm}$, and the resulting Sb particles were separated from the stainless-steel balls.

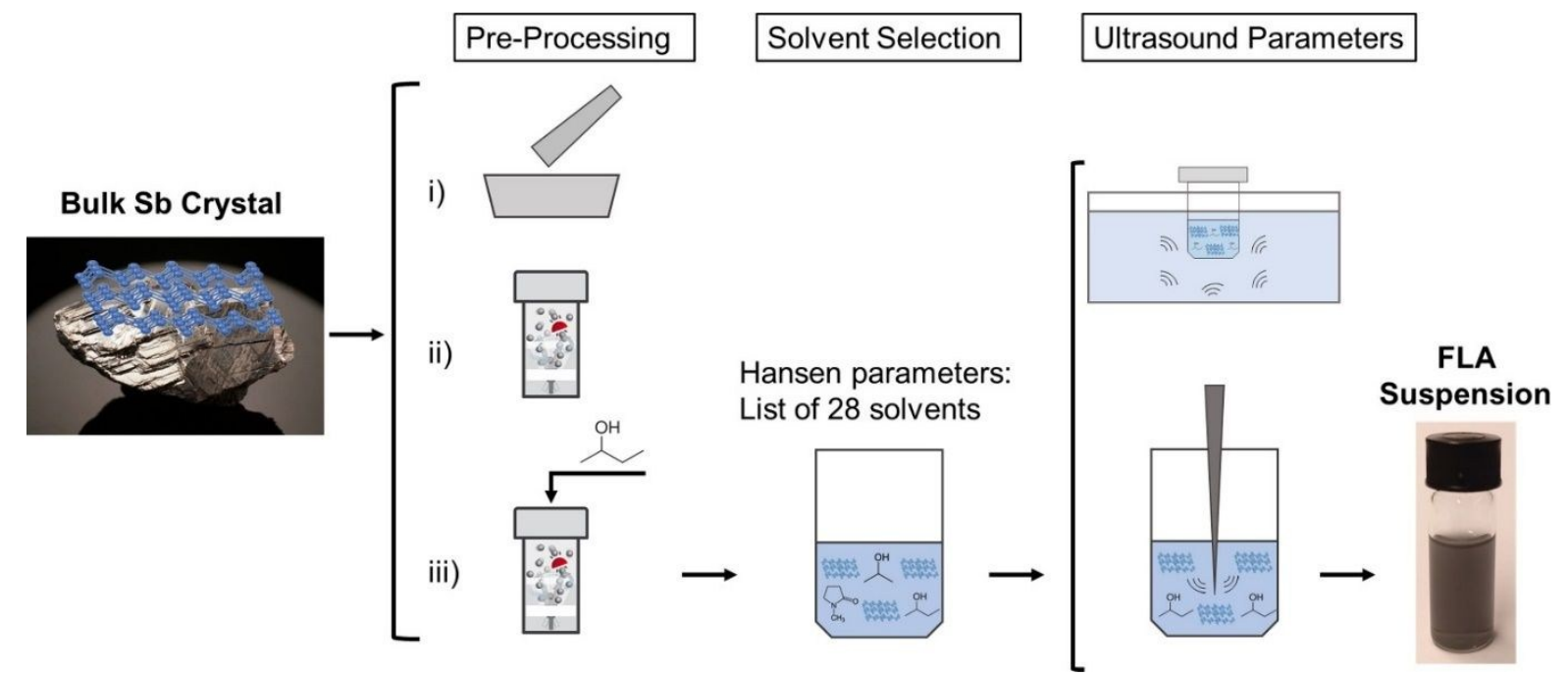

Fig 1. Summary of the strategy followed to optimize the liquid phase exfoliation process of bulk antimony, involving different enchained steps. Firstly, the pre-processing using different approaches: i) grinding the bulk Sb crystals, ii) dry ball-milling the bulk Sb crystals, and iii) wet ball-milling the bulk Sb crystals. Secondly, a solvent selection based on the Hansen parameters using 28 different solvents, and thirdly, a comparison of ultrasound parameters, involving bath and tip sonication. The arrows highlight the optimum route for obtaining few-layers antimonene with the highest concentration. The optical image shows a typical highly concentrated FL antimonene suspension. 
After the grinding process, a stainless-steel reactor with a volume of $5 \mathrm{~mL}$ (Retsch 1.4112) was filled under ambient conditions with $300 \mathrm{mg}$ of grinded Sb powder, 3 stainless steel balls of $4.74 \mathrm{~mm}$ diameter and $0.5 \mathrm{~mL}$ of butan-2-ol $(99.5 \%$, Sigma Aldrich). Subsequently, the samples were milled for 120 min. at $30 \mathrm{~Hz}$ in a Retsch MM 400 mixer mill. After milling, the reactors were washed with butan-2-ol to obtain all of the grey metallic Sb paste, which was then centrifuged at $13000 \mathrm{rpm}$ for $30 \mathrm{~min}$. The sedimented $\mathrm{Sb}$ was dried on a hot plate at $100 \stackrel{\circ}{\circ}$ for 12 hours and another 24 hours in the drying oven at 75 oC and a few mbar.

\section{Tip Sonication of Sb Crystals}

$10 \mathrm{mg}$ of "wet ball-milled" Sb crystals were put on a $20 \mathrm{~mL}$ vial with $10 \mathrm{~mL}$ of solvent. The mixture was sonicated for $40 \mathrm{~min}$. at $400 \mathrm{~W}$ and $24 \mathrm{kHz}$ delivering the ultrasound power in pulses 0.5 $\mathrm{s}$ long every $1 \mathrm{~s}$. Then, the resulting black suspension was centrifuged at $3000 \mathrm{rpm}$ (746 g) for $3 \mathrm{~min}$, in order to eliminate the unexfoliated crystals, and the clear supernatant was recovered. $20 \mu \mathrm{L}$ of the suspension were casted on a $\mathrm{SiO}_{2}$ surface and dried under an argon flow before drying completely. Sonication was performed using a Hielscher UP400S ultrasonic processor equipped with a $3 \mathrm{~mm}$ sonotrode. Centrifugation was carried out in a MPW-350R centrifuge using $2 \mathrm{~mL}$ Eppendorf.

\section{Bath Sonication of Sb Crystals}

$10 \mathrm{mg}$ of "wet ball-milled" Sb crystals was put on a $20 \mathrm{~mL}$ vial with $10 \mathrm{~mL}$ of solvent. The mixture was sonicated for $40 \mathrm{~min}$ in the ultrasonic bath at $380 \mathrm{~W}$ and $37 \mathrm{kHz}$. Then, the resulting black suspension was centrifuged at $3000 \mathrm{rpm}$ (746 g) for $3 \mathrm{~min}$, in order to eliminate the unexfoliated crystals, and the clear supernatant was recovered. $20 \mu \mathrm{L}$ of the suspension were casted on a $\mathrm{SiO}_{2}$ surface and dried under an argon flow before drying completely. Sonication was performed using an Elmasonic $\mathrm{P} 300 \mathrm{H}$ ultrasonic bath. Centrifugation was carried out in a MPW-350R centrifuge using $2 \mathrm{~mL}$ Eppendorf.

\section{Materials characterization}

Turbidity measurements were carried out using a HI-88713 Bench Top Turbidity Meter Hanna Instruments. To change between turbidity measurements to concentration values was needed to make a calibration (†Fig.S1). The real value of concentration to make the calibration was obtained by vacuum drying overnight one FL antimonene sample and the obtained solid was weighted to know exactly the amount of Sb that was in the sample AFM measurements were carried out using a Cervantes Fullmode AFM from Nanotec Electronica SL. WSxM software (www. wsxmsolutions.com) was employed both for data acquisition and image processing ${ }^{31}$. PPP-NCHR cantilevers (nanosensors.com) with a nominal spring constant of $42 \mathrm{~N} / \mathrm{m}$ and tip radius of less than $7 \mathrm{~nm}$ were employed. Tapping mode were used for imaging to ensure that the nanolayers would not be damaged by the tip ${ }^{32}$. Raman spectra were acquired on a LabRam HR Evolution confocal Raman microscope (Horiba) equipped with an automated $\mathrm{XYZ}$ table using $0.80 \mathrm{NA}$ objectives. All measurements were conducted ussingonap excitation wavelength of $532 \mathrm{~nm}$, with aRakqueisiż $\mathrm{s}$ and a grating of 1800 grooves $/ \mathrm{mm}$. To minimize the photoinduced laser oxidation of the samples, the laser intensity was kept at $10 \%(1.6 \mathrm{~mW})$. The step sizes in the Raman mappings were in the $0.2-0.5 \mu \mathrm{m}$ range depending on the experiments. Data processing was performed using Lab Spec 5 as evaluation software. When extracting mean intensities of individual $\mathrm{Sb}$ Raman modes, it is important to keep each spectral range constant, e.g. from $90-110 \mathrm{~cm}^{-1}$ and from $140-160 \mathrm{~cm}^{-1}$ because otherwise the resulting value of the $E_{g} / A_{1 g}$ - ratio can be slightly influenced. TEM images were obtained in a JEOL JEM 2100 FX TEM system with an accelerating voltage of $200 \mathrm{kV}$. The microscope has a multiscan charge-coupled device (CCD) camera ORIUS SC1000 and an OXFORD INCA X-Ray Energy Dispersive Spectroscopy (XEDS) microanalysis system. SEM analysis was performed using a Philips XL 30 S-FEG microscope operating at an accelerating voltage of $10 \mathrm{kV}$. X-ray diffraction patterns were measured on a Bruker D8 Advance with $\mathrm{Cu} \mathrm{K \alpha}$ radiation with rapid detector (lynxeye).

\section{Electrochemical characterization}

For electrode preparation, several aliquots of $50 \mu \mathrm{L}$ were drop casted in a previously polished (sequentially with 1.0, 0.3 and $0.05 \mu \mathrm{m}$ alumina powder) $3 \mathrm{~mm}$ Glassy Carbon (GC) electrode reaching a total mass loading of about $0.18 \mathrm{mg} \cdot \mathrm{cm}^{-2}$. Further, 4 $\mu \mathrm{L}$ of Nafion solution (5\%) were added. Solvents were let evaporated at room temperature. The electrochemical measurements were carried out in a typical three electrode cell using modified GC electrode as the working electrode, a platinum wire as the counter and a silver-silver chloride electrode $(\mathrm{Ag} / \mathrm{AgCl}(3 \mathrm{M}))$ as reference. All potentials were converted to the reversible hydrogen electrode (RHE). Measurements were performed using an Autolab PGSTAT 128N potentiostat/galvanostat. To analyse the electrocatalytical performance of the samples, linear sweep voltammetry (LSV) measurements were carried out at $5 \mathrm{mV} \cdot \mathrm{s}^{-1}$ in a previously $\mathrm{N}_{2}$ purged $0.5 \mathrm{M} \mathrm{H}_{2} \mathrm{SO}_{4}$ aqueous solution. Cyclic voltammetry (CV) curves were recorded before and after LSV measurements, at $100 \mathrm{mV} \cdot \mathrm{s}^{-1}$ scan rate between -0.6 and $0.6 \mathrm{~V}$ vs. $\mathrm{Ag} / \mathrm{AgCl}(3 \mathrm{M})$. To further investigate the electrochemical behaviour, CVs were recorded at $50 \mathrm{mV} \cdot \mathrm{s}^{-1}$, fixing the cathodic/anodic potential and varying the anodic/cathodic limit, respectively. The charge (Q) was estimated using the following formula:

$$
Q=\int_{t_{o}}^{t_{f}} I \cdot d t
$$

\section{X-Ray Photoelectron (XPS) measurements}

XPS experiments were performed in an Ultra-High Vacuum chamber. $\mathrm{Mg} \mathrm{K \alpha}$ radiation excites core level photoelectrons, which are detected using a Specs Phoibos-150 electron analyzer with a constant pass energy of $20 \mathrm{eV}$. The core level binding energies were calibrated using as references the binding energies of $C 1 s$ and $A u 4 f$ in contact with the sample. The line shape of core levels was fitted using a Shirley background and asymmetric singlet pseudo-Voigt functions. 
The fit was optimized using a Levenberg-Marquardt algorithm with a routine running in IGOR Pro (WaveMatrix Inc.). ${ }^{33}$ The quality of the fit was judged from a reliability factor, the normalized $\chi^{2}$.

\section{Results and discussion}

Fig.1 summarized our strategy to optimize the formation of concentrated suspensions containing high-quality FL antimonene nanolayers with a high DA ratio.

\section{Pre-processing of bulk Sb crystals}

One issue to be solved regarding the LPE of Sb crystals is the low concentration of the final suspensions. In order to enhance the FL antimonene concentration, a fundamental key is the way to process the bulk Sb crystals before the LPE process starts, by reducing the particle size and the thickness of the starting crystallites. Thus, in this first step, the goal is to analyse the effect of the initial particle size of Sb crystals in the final concentration of FL antimonene suspensions. In this section, we have analysed three different ways of pre-processing bulk Sb crystals and the concentration of the suspensions formed after LPE. DOI: 10.1039/C9TA06072C

The three selected approaches are based on previous investigation in this field: ${ }^{18,24,25,34,35}$ i) grinding the bulk $\mathrm{Sb}$ crystals, ii) dry ball-milling the bulk Sb crystals, and iii) wet ballmilling the bulk Sb crystals. For all of these experiments we used as reference LPE conditions to analyse the final concentration of $\mathrm{FL}$ antimonene suspensions: initial Sb concentration of 0.1 $\mathrm{g} \cdot \mathrm{L}^{-1}$, tip sonication for $40 \mathrm{~min}$. at $400 \mathrm{~W}$ and $24 \mathrm{kHz}$ with ultrasound power in pulses $0.5 \mathrm{~s}$ long every $1 \mathrm{~s}$ and centrifugation at $3000 \mathrm{rpm}$ for $1 \mathrm{~min} .{ }^{18}$

The three pre-processed Sb crystals were characterized by X-Ray Powder Diffraction (XRPD) and Scanning Electron Microscopy (SEM), in order to analyse its structure and morphology, respectively. In Fig.2a it is depicted the corresponding XRPD patterns of the three pre-processed $\mathrm{Sb}$ crystals, showing the typical crystalline structure of $\mathrm{Sb}$. The obtained pre-processed $\mathrm{Sb}$ crystals could be classified as a rhombohedral structure, $\beta$-phase. There are no new peaks, discarding the appearance of side species such as antimony oxides. a)

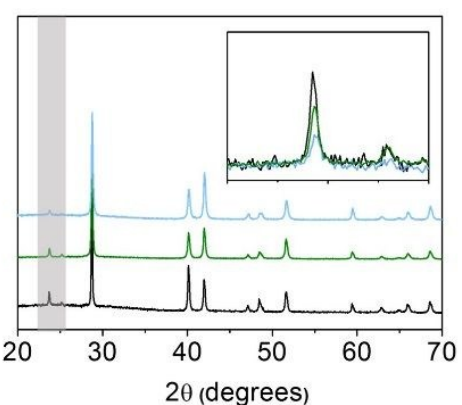

d)

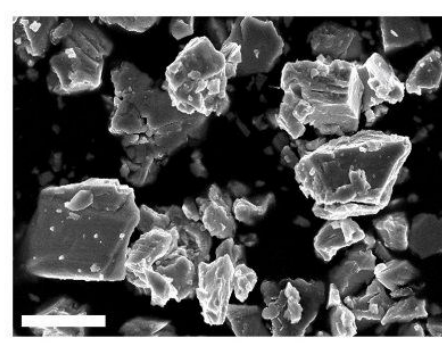

g)

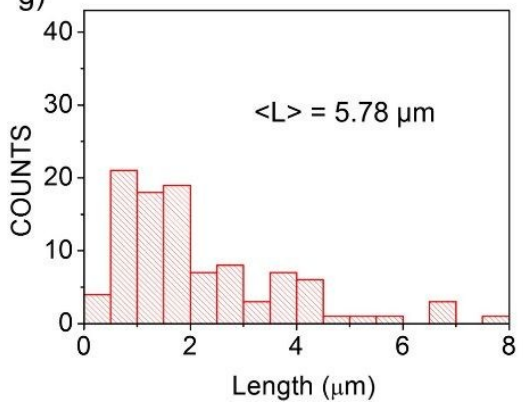

b)

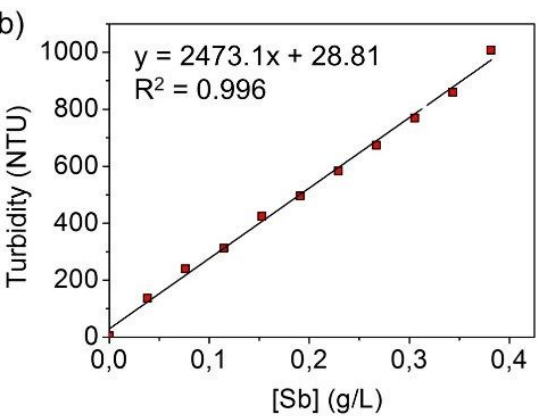

e)

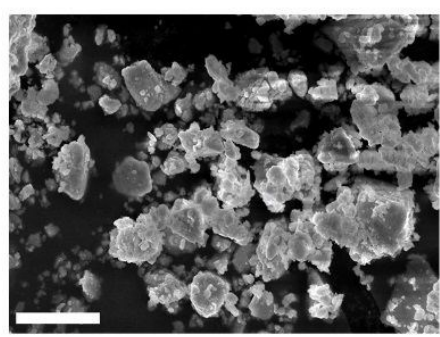

h)

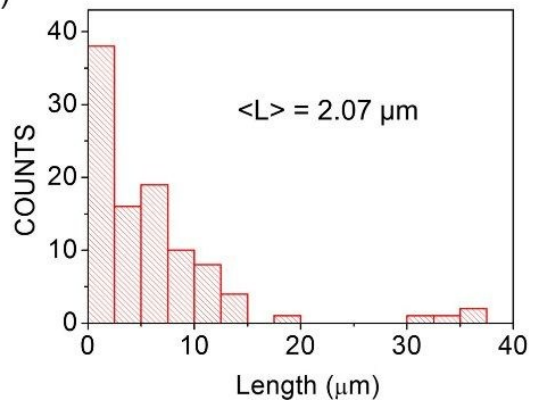

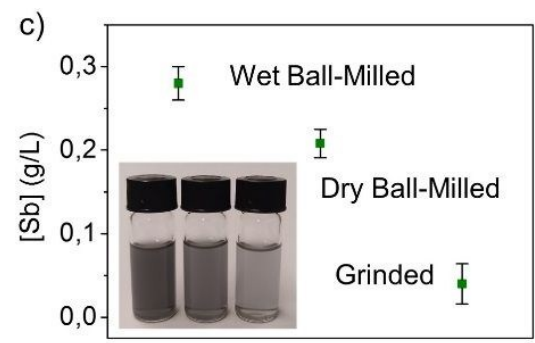

f)

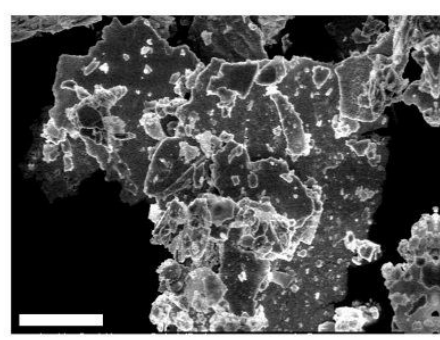

i)

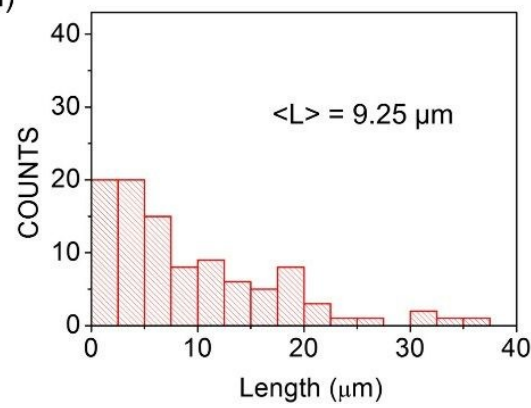

Fig 2. a) PXRD patterns of grinded Sb crystals (black), dry ball-milled Sb crystals (green) and wet ball-milled Sb crystals (blue). The inset shows a magnification of the area coloured in grey in Fig.1a. b) Calibration of the turbidity (NTU units) versus concentration ( $\mathrm{g} \cdot \mathrm{L}^{-1}$ ). c) Concentration of the samples using different starting materials. The inset displays a photograph of the three different suspensions with a clear change of colour due to change on the concentration. SEM images (scale bar of the SEM images equal to $5 \mu \mathrm{m}$ ) of: $\mathrm{d}$ ) grinded Sb, e) dry ball-milled Sb, and f) wet ball-milled Sb crystals. Length histograms of the corresponding SEM images in $\mu \mathrm{m}$ of: g) grinded Sb, h) dry ball-milled Sb, and f) wet ball-milled Sb crystals. 
Fig.2a confirms the absence of significant changes between the three XRPD patterns, meaning that these processes do not affect the in-plane crystalline structure of $\mathrm{Sb}$. The only noticeable change between the three XRPD patterns is the relative diminishing of the peak at 23.70 corresponding to the (003) facet, indicative of a greater degree of exfoliation along the $c$-axis in the wet ball-milled sample compared to the other two (see Fig.2a, inset).

The morphology of the three pre-processed Sb crystals was analysed by SEM, making length histograms for the three samples and showing clear differences between the processes. In the case of grinded Sb crystals (Fig.2d), the edges seem to be sharper than in the other two cases, exhibiting lateral sizes of ca. $5.78 \mu \mathrm{m}$. The dry ball-milled Sb crystals (Fig.2e) images show undefined edges and smaller sizes ( $c a .2 .07 \mu \mathrm{m}$ ) compared to the grinded ones, suggesting an excessive downsizing of the particles. Remarkably, the one obtained by wet ball-milling in 2butanol (Fig.2f) has the crystals with larger lateral dimensions (ca. $9.25 \mu \mathrm{m}$ ) and also smaller thicknesses. After having analysed the crystal structure and morphology of the three preprocessed Sb crystals, it seems that none of the processes induce changes neither in the crystal structure nor in composition, while the particle size of the Sb crystals changes from one to another. In order to choose the best option between the different processes, the three crystals were used as starting material to generate suspensions in 2-butanol by sonication, and then the final FL antimonene concentration was checked. The concentration values were obtained by measuring the turbidity of the samples, which is directly related to the concentration (Fig. $2 \mathrm{~b}$ and Experimental Section for details). Fig.2c shows that the most concentrated sample is the one that used the powder obtained by wet ball-milling as starting material. Therefore, the most suitable pre-processing approach in order to enhance the concentration of $\mathrm{FL}$ antimonene suspensions is the wet ball-milling of Sb crystals using 2butanol.

\section{Solvent selection}

It is well established that the solvent used to exfoliate layered materials is probably the most critical parameter during the LPE process. ${ }^{36}$ The aim of this section is to analyse the influence of the solvent used in the LPE of Sb crystals in both, the final concentration of $\mathrm{FL}$ antimonene suspensions and the DA ratio. In order to increase either the concentration and/or the DA ratio of the samples, a survey of 28 pure solvents and mixtures of them with water has been tested. All the experiments were done using as starting material wet ball-milled Sb crystals in 2butanol, optimized in the previous section. In these experiments, we have fixed the sonication time and the centrifugation parameters according to our previous LPE results. ${ }^{18}$ Therefore, we used for all the experiments $40 \mathrm{~min}$ of sonication, and $3000 \mathrm{rpm}$ for $1 \mathrm{~min}$ of centrifugation.

On the other hand, it is well established that the concentration of the suspensions is maximized when the energy cost of the exfoliation process is minimized, i.e. when the surface energy of the solvent matches with the surfaceienergy of the layered crystals, as shown in eq. (19. OI: 10.1039/C9TA06072C

$$
\frac{\Delta H_{\text {mix }}}{V_{\text {mix }}} \approx \frac{2}{T_{\text {layer }}}\left(\delta_{\text {layer }}-\delta_{\text {solvent }}\right)^{2} \emptyset \quad \text { eq. (1) }
$$

Where $\delta_{i}=\sqrt{E_{\text {sur }}^{i}}$ the square root of the component surface energy, $T_{\text {layer }}$ is the thickness of an antimonene nanolayer and $\varnothing$ is the volume fraction.

Typical solvents used in LPE have surface tension values within a well-defined range, ${ }^{30,36-38}$ therefore we need to calculate the surface energy of antimony in order to select the most suitable solvents. FFig.S1 shows the results of the theoretical calculations we have carried out for obtaining the surface energy of a Sb (111) crystal surface, leading to a value of $148.8 \mathrm{~mJ} \cdot \mathrm{m}^{-2}$. This calculated value is overestimated since it is obtained under ideal static conditions which do not consider the dynamics of the LPE process (see tESI.2 for more information). ${ }^{39}$ In our initial survey we have chosen a selection of solvents with a wide variety of surface tensions, ${ }^{30}$ but intentionally included some solvents known to be good dispersants for layered materials and surfactants to complete the total survey of 28 solvents (see TTable S1). The experiments have been run by triplicate and the concentration represents the averages. FFig.S2a collects the concentration values of $\mathrm{FL}$ antimonene obtained after sonication and centrifugation (40 min of tip sonication, and $3000 \mathrm{rpm}$ for $1 \mathrm{~min}$ of centrifugation) as a function of the surface tension of each solvent. The concentration values of the suspensions increase for those solvents with surface tensions in the range of $23-42 \mathrm{~mJ} \cdot \mathrm{m}^{-2}$, meaning surface energy values in the range $52-71 \mathrm{~mJ} \cdot \mathrm{m}^{-2}$. These values are relatively close to the surface energy value calculated for Sb (ca. $149 \mathrm{~mJ} \cdot \mathrm{m}^{-2}$ ).

However, †Fig.S2a shows also some solvents with surface tension values within the range suitable for the exfoliation of antimonene, but yielded a rather low concentrated suspension. This is commonly observed in the LPE of other layered materials and it often indicates that other factors need to be considered in order to rationalize the effect of the solvent on the exfoliation of our material. ${ }^{26,40}$ Those factors should consider the solutesolvents molecular interactions, namely dispersion forces $\left(\delta_{D}\right)$, polar interactions $\left(\delta_{\mathrm{P}}\right)$, and specific interactions such as hydrogen bonding $\delta_{\mathrm{H}}$. In this regard, we used both the Hildebrand $\left(\delta_{T}\right)$ and Hansen's solubility parameters that account for all the involved molecular interactions as criterion to assess the suitability of the investigated solvents for the exfoliation of antimonene (see +ESI.1 for details).

†Fig.S2b depicts a defined peak of $\mathrm{FL}$ antimonene concentration between $22-30 \mathrm{MPa}^{1 / 2}$ for the $\delta_{\mathrm{T}}$ parameter. However, clearly $\delta_{T}$ parameter cannot be considered independently as the key parameter, because some solvents showing similar $\delta_{\mathrm{T}}$ values present low concentration values. Therefore, Hildebrand parameter is too rough to fully describe the exfoliation/dispersion process. †Fig.S2c-e displays Hansen's model parameters versus concentration in which a defined peak of $\mathrm{FL}$ antimonene concentration at $c a .17 \mathrm{MPa}^{1 / 2}$ for $\delta_{\mathrm{D}}$ and 7$22 \mathrm{MPa}^{1 / 2}$ for $\delta_{\mathrm{P}}$ and $\delta_{\mathrm{H}}$ can be observed. These results clearly show that the best solvents to produce high concentration of $\mathrm{FL}$ 
antimonene match with these sets of Hansen solubility parameters (†Table S2).

a)

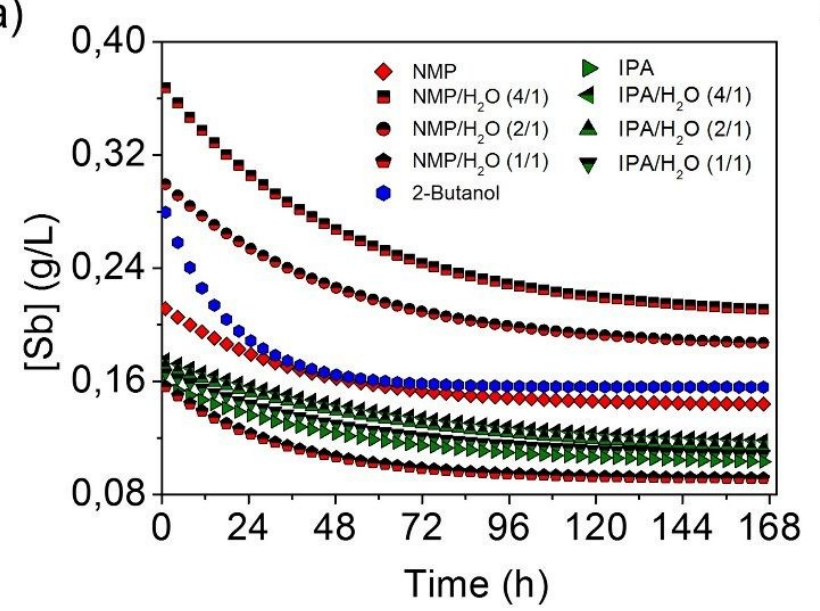

b)

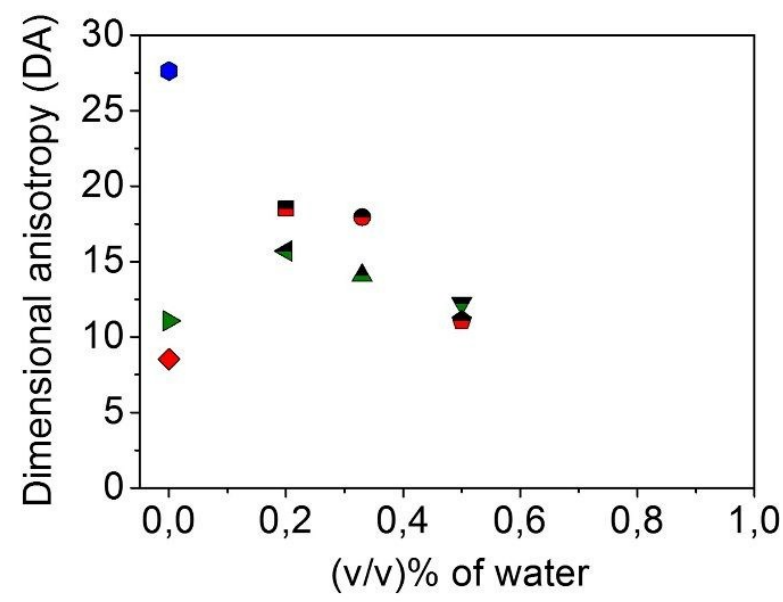

Fig 3. a) Concentration of FL antimonene in suspension after centrifugation, [Sb] $\left(\mathrm{g} \cdot \mathrm{L}^{-1}\right)$, plotted versus sedimentation time of the samples prepared with the solvents described in the legend. b) Plot of the DA ratio of the FL antimonene nanolayers as a function of the volume fraction (\%) of water in the solvent mixtures showed in Fig.3a.

It is remarkable the case of $\mathrm{NMP} / \mathrm{H}_{2} \mathrm{O}(4: 1)$ that gives rise a concentration of $0.368 \mathrm{~g} \cdot \mathrm{L}^{-1}$, what means an approximate yield of 37 wt\%, which is $c a .30$ times higher than the highest previously reported for LPE of antimony crystals (ca. $0.014 \mathrm{~g} \cdot \mathrm{L}-$ 1). ${ }^{24}$ Even though the obtained yield is the highest reported so far for antimonene, in case we want to scale-up the process for industrial application is necessary to rise greater values. For that reason, we recycled the sediment obtained after centrifugation to the first step of the LPE process, obtaining almost the same yield values for each cycle, up to a maximum of 7 cycles. A total of $8.94 \mathrm{mg}$ of antimonene was finally exfoliated what means that the final yield of the whole process is $\sim 90 \mathrm{wt} \%$ after recycling the sediment for 7 times. It is also worth mentioning that the adjustment of the ratio between the organic solvent and water in the mixtures determines the optimal concentration. Thus, the 4:1 and 2:1 proportions of solvent/water mixtures, in NMP case, match well with the set of Hansen solubility parameters described above and in consequence give higher concentration (†Fig.S2c-e).

Therefore, the solvents that give rise to the highest concentration values are NMP, IPA and 2-butanol, and some mixtures of them with water. This result is consequence of two factors: i) the rationally matching of the solvents with the surface energy of antimony and, even more remarkably, ii) with the set of Hansen solubility parameters. It is obvious that another important limiting factor determining the potential use of the antimonene suspensions for several applications is their stability with time, i.e. particle aggregation/sedimentation. Therefore, we tested the samples with the higher FL antimonene concentration from the initial survey and measured their concentration decay at different times $(1,24,48$ and $168 \mathrm{~h}$ ). Fig.3a demonstrates that most of the suspensions evaluated undergo a concentration decrease of 10-20\% and ca. $50 \%$ upon standing the suspension at 20 oC for 1 or 2 days, respectively, and almost became stable for weeks after 2 days. In terms of suspensions usability, it seems a reasonably good result for most of the applications, but it is even better because we have observed that after long times of sedimentation, the suspensions recover their initial concentration values by resonicating them for just $5 \mathrm{~min}$.

Another important issue is the effect caused in the concentration of the suspension with the solvent/water ratio. Fig.2a shows that for $\mathrm{NMP} / \mathrm{H}_{2} \mathrm{O}, 4: 1$ and $2: 1$ ratios rend to optimum values of concentration, but in the case of IPA/ $\mathrm{H}_{2} \mathrm{O}$ the differences between using diverse ratios are almost neglectable.

The morphology of the nanolayers obtained in the suspensions has been evaluated using atomic force microscopy (AFM). +Fig.S3-S5 show a statistical AFM analysis for the dropcasted suspensions on $\mathrm{SiO}_{2}$ with the highest antimonene concentration, based on histograms over 150 antimonene nanolayers for each sample. The histograms show that the mean length, $<L>$, of most of $\mathrm{FL}$ antimonene nanolayers contained in the samples is $\sim 300 \mathrm{~nm}$ while the mean height, $\langle\mathrm{H}\rangle$, is $\sim 20 \mathrm{~nm}$. It has to be considered that when using AFM we are evaluating the apparent thickness, which overestimates the real nanolayer height due to the adhesion and capillary forces involved. ${ }^{32} \mathrm{~A}$ closer examination of the LPE results collected in †Fig.S3-S5 show a limiting minimum height of $c a$. 4-6 nm using 2-butanol as solvent, which is associated with a maximum length of $c a .320 \mathrm{~nm}$. However, the most typical apparent heights obtained for those solvents providing good concentration values are in the range of $15-22 \mathrm{~nm}$ while their length almost shows similar values, ca. 250-375 $\mathrm{nm}$. The highest DA ratio (Fig.2b) corresponds to the nanolayers isolated from the suspensions obtained using 2-butanol as solvent (DA ca. 27.6), while for the samples prepared with IPA and NMP there is an optimal proportion of the solvent/water mixture at $4: 1$, resulting in DA ratios of $c a .15 .7$ and $c a .18 .5$, respectively.

Therefore, considering all the factors so far analysed we observe that the best results in terms of concentration, correspond to those obtained using as exfoliating solvent a 
mixture of 4:1 NMP/ $\mathrm{H}_{2} \mathrm{O}$, with a final concentration of $0.368 \mathrm{gL}^{-1}$ and a DA ratio of $c a$. 18.52. However, 2-butanol seems to be the most suitable solvent because displays an excellent compromise between concentration $\left(0.279 \mathrm{~g} \cdot \mathrm{L}^{-1}\right)$ and $\mathrm{DA}$, satio (ca. 27.6).
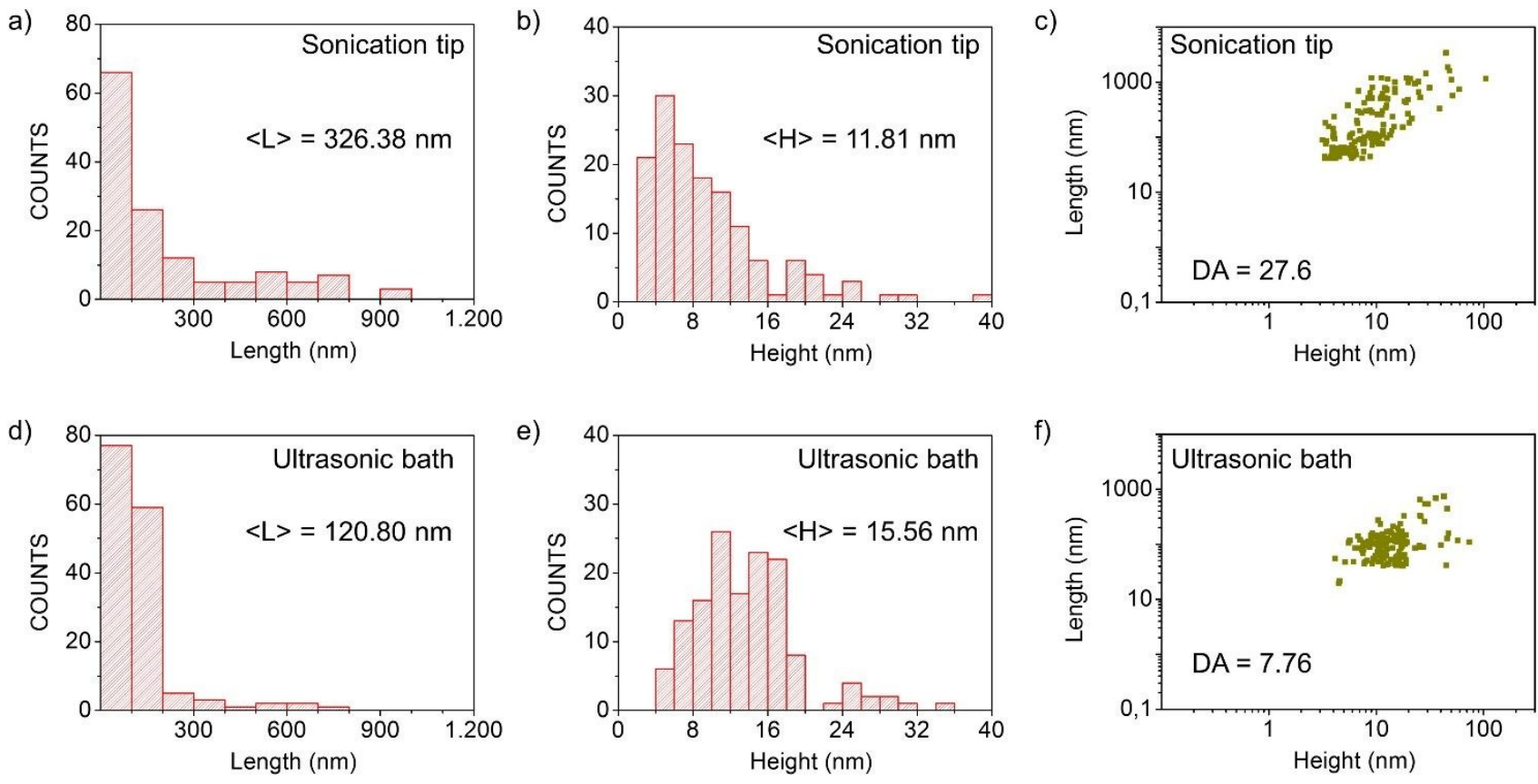

Fig 4. Height histogram, length histogram and plot of the length as a function of the height of the FL antimonene nanolayers contained in the samples prepared using different devices: a), b), c) sonication tip and d), e), f) ultrasonic bath.

\section{Ultrasound parameters}

Finally, in order to improve the LPE process we have evaluated the effects on the concentration and DA ratio of the FL antimonene suspensions using different ultrasound techniques: sonication tip and ultrasonic bath.

It is well known that for layered inorganic materials, tip sonication is preferred over bath sonication due to the higher production rates. However, bath sonication may offer a lowercost alternative to tip sonication and a lower energy input into the sample, as it is less localized. With this technique, longer processing times are required, compared to tip sonication, to achieve an equivalent concentration of dispersed material and, in general, the process is less reproducible. ${ }^{37}$

For this analysis we have used as starting material, wet ballmilled Sb crystals, 2-butanol as LPE solvent, 40 min of sonication and $3000 \mathrm{rpm}$ for $1 \mathrm{~min}$ for centrifugation. The results show that using ultrasonic tip the obtained concentration $\left(\sim 0.289 \mathrm{~g} \cdot \mathrm{L}^{-1}\right)$, is higher than the one obtained using ultrasonic bath ( $\left.0.262 \mathrm{~g} \cdot \mathrm{L}^{-1}\right)$. Fig.4 displays the DA ratio for both samples from the mean statistic values of morphology, obtaining a much lower DA ratio using the ultrasonic-bath (ca. 7.8) than using the sonication tip (ca. 27.6). The lower DA ratio means a decrease in the lateral dimensions of the nanolayers while they show similar thicknesses (Fig.4). Both results can be rationalized considering the experimental differences between both techniques. Thus, while the sonication tip approach delivers energy to the dispersion medium directly, when using ultrasonic bath the energy needs to go through the solvent medium and the flask where is the material to exfoliate it. ${ }^{37}$
Finally, we have also tested the effect of the ultrasonic wave amplitude of the sonication tip. +Fig.S6 show that the higher the amplitude, the higher the concentration: $[\mathrm{Sb}]_{100 \%}=0.289 \mathrm{~g} \cdot \mathrm{L}^{-1}$; $[\mathrm{Sb}]_{50 \%}=0.274 \mathrm{~g} \cdot \mathrm{L}^{-1}$ and $[\mathrm{Sb}]_{20 \%}=0.252 \mathrm{~g} \cdot \mathrm{L}^{-1}$. Moreover, the DA also increases with the amplitude value: $\mathrm{DA}_{100 \%}=27.6 ; \mathrm{DA}_{50 \%}=$ 23.2; and $\mathrm{DA}_{20 \%}=19.9$. Therefore, $100 \%$ amplitude yields the best results in terms of concentration and DA.

\section{FL antimonene nanolayers characterization}

The energy used to assist the exfoliation in the LPE process can affect the 2D material quality generating atomic defects in the structure of the nanolayer or inducing some chemical changes (e.g. partial oxidation). In this section we have characterized the $\mathrm{FL}$ antimonene nanolayers isolated under the LPE optimal experimental conditions -i.e.: wet ball-milled Sb crystals in 2butanol as starting material; 2-butanol as LPE solvent with 40 min of sonication and $3000 \mathrm{rpm}$ for $1 \mathrm{~min}$ for centrifugation using a sonicator tip at $100 \%$ amplitude- by means of AFM, Raman and TEM.

Fig. 5 shows the characterization of a representative sample prepared using the aforementioned optimal conditions, showing the highest DA value. The dispersions observed in an optical image show an intense turbidity with a characteristic grey colour, indicative of high concentration. AFM analysis of samples deposited on $\mathrm{SiO}_{2} / \mathrm{Si}$ substrates exhibits particles with a minimum thickness of $4 \mathrm{~nm}$ and average lateral dimensions of ca. $250-375 \mathrm{~nm}$, as previously commented. To further characterize the quality of the sample, we have performed scanning Raman microscopy (SRM) mappings (>14,000 single 
point spectra) immediately after the exfoliation and correlated the results with AFM.

a)

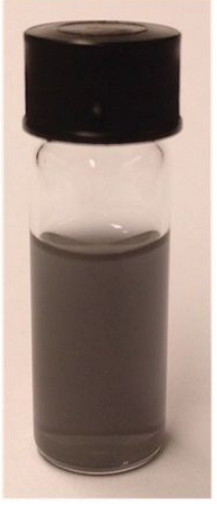

b)

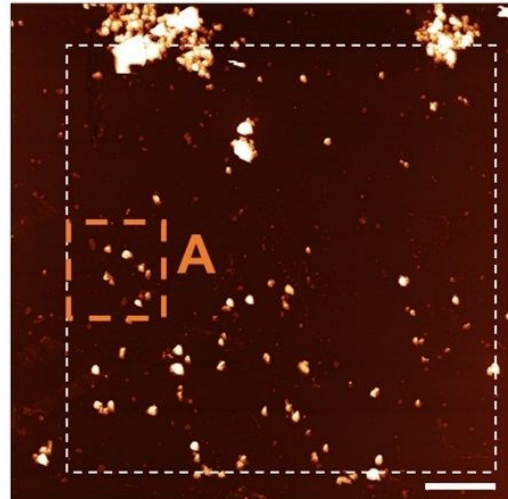

View Article Online DOI: $10.1039 /$ C9TA06072C d)

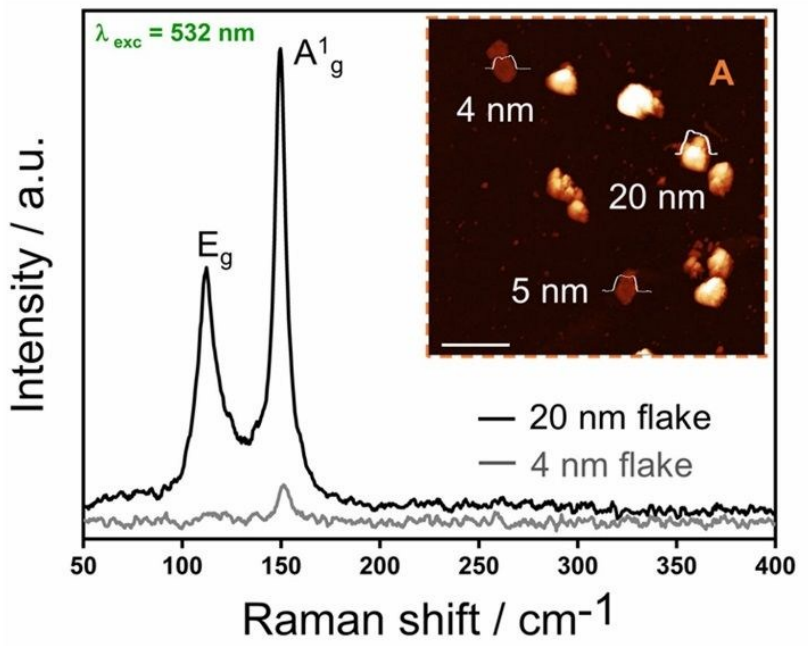

c)

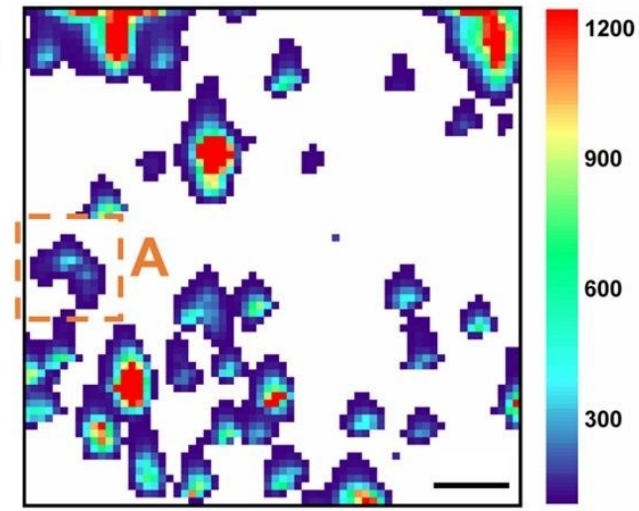

e)

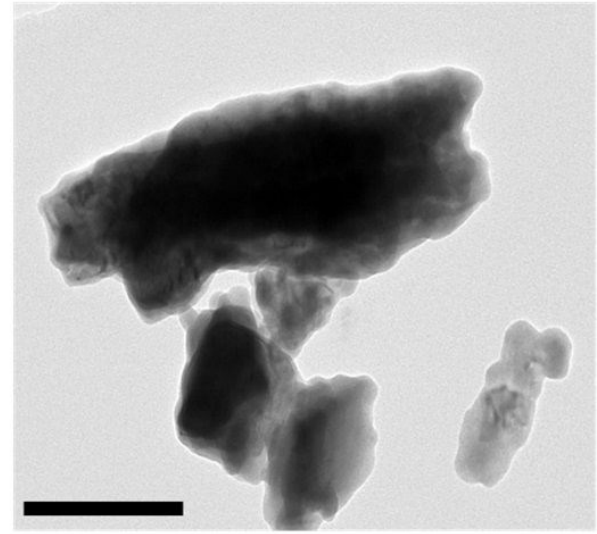

Fig 5. a) Optical image of the FL antimonene suspension prepared using 2-butanol as a solvent. b) Representative topographic AFM image (scale bar equal to $2 \mu \mathrm{m}$ ) of the exfoliated FL antimonene nanolayers contained in the suspension in (a). c) Corresponding Raman $\mathrm{A}_{1 \mathrm{~g}}\left(\lambda_{\text {exc }}=532 \mathrm{~nm}\right.$ ) mapping (scale bar equal to $2 \mu \mathrm{m}$ ) of the exfoliated FL antimonene nanolayers contained in the area dotted in white in (b) (> 14000 single point spectra over a surface area of $13 \mu \mathrm{m}^{2}$ using a step size of $0.2 \mu \mathrm{m}$ ). d) Single-point spectra measured at different thicknesses according to the topographic AFM image (inset scale bar equal to $500 \mathrm{~nm}$ ) of the small area (A) dotted in orange in (a) and (b) showing the height profiles of ca. 4,5 and $20 \mathrm{~nm}$ respectively. e) TEM image (scale bar equal to $100 \mathrm{~nm}$ ) of FL antimonene nanolayers contained in the suspension in (a).

Fig. $5 \mathrm{~d}$ shows the single point spectra for a FL antimonene of $c a$. $20 \mathrm{~nm}$ of thickness revealing the representative main phonon peaks, the $A_{1 \mathrm{~g}}$ mode at $149.8 \mathrm{~cm}^{-1}$ and $E_{\mathrm{g}}$ mode at $110.0 \mathrm{~cm}^{-1}$. The single point spectra obtained from the pixel associated to the ca. $4 \mathrm{~nm}$ flake, which is close to the diffraction limit of our Raman spectrophotometer, suggest a phonon relaxing effect when the sample thickness decreases, in good agreement with theoretical predictions and recent reports (Fig.5d). ${ }^{15,41}$ The correlation between SRM and AFM shown in Fig.5b-c represents the state-of-the-art of an antimonene nanolayer prepared by LPE with the smaller thickness so far reported. ${ }^{15,18}$ Even though all the FL antimonene nanolayers shown in Fig. $5 \mathrm{~b}$ can be easily correlated with the $A^{1}$ gignals from Fig. $5 c$, it is worth mentioning that the resolution of the mapping in some cases has a broadening in the signal that can be attributed to nanolayer aggregation, according to AFM images. The crumpled morphology observed by AFM has been further confirmed by transmission electron microscopy (TEM) measurements, showing lateral dimensions of $\sim 300 \mathrm{~nm}$ (Fig. 5e). Selected area
X-Ray energy dispersive spectroscopy (XEDS) measurements corroborated their composition, showing small signals of oxygen (Fig. S7). Indeed, a careful examination of the Raman mappings reveals that for some flakes exhibiting high intensity, it is possible to detect some Raman signatures attributed to antimony oxides, $c a .255 \mathrm{~cm}^{-1}$, tentatively related to the $A_{g}$ mode of $\mathrm{Sb}_{2} \mathrm{O}_{3}$.

In order to corroborate the oxidation, we have investigated this sample by X-ray photoelectron spectroscopy (XPS) analysis, showing weak $3 d_{3 / 2,5 / 2}$ signals of antimony in oxidation state zero at $528.5 \mathrm{eV}$ for the $3 d_{5 / 2}$ level, along with significant contributions from $\mathrm{Sb}$ in higher oxidation states (mainly $\mathrm{Sb}_{2} \mathrm{O}_{3}$ ) at around $530.9 \mathrm{eV}$ (Fig.6, see also ESI.3). Moreover, among the solvents showing optimal Hansen parameters investigated in this work, NMP has been reported as an excellent protective solvent against oxidation for 2D materials. Along this front, other 2D-pnictogens such as black phosphorus has been successfully passivized with NMP for several days under environmental conditions. ${ }^{13,42,43}$ Therefore, we have 
investigated this sample by XPS to reveal if we have an analogous effect in antimonene.

Similarly to the ionic-liquid protected few-layer antimonene, ${ }^{25}$ next to the broad $\mathrm{O} 1 \mathrm{~s}$ signal at $532.5 \mathrm{eV}$ from the surface enriched NMP contamination, the $\mathrm{Sb} 3 \mathrm{~d}$ region between 526 and $546 \mathrm{eV}$ of the Sb-NMP sample reveals very weak $\mathrm{Sb} 3 d_{3 / 2,5 / 2}$ signals at slightly lower binding energies (see inset in Fig.6). These results suggest a less oxidized antimonene and, therefore, the formation of a protective NMP layer a fewnanometers thick on the surface of the antimonene flakes.

Last but not least, in order to further investigate the effect of this superficial oxidation on the properties of LPE antimonene, we took advantage of the high sensitivity of electrochemical measurements towards oxidation. Specifically, we have studied the electrochemical behaviour of the FL antimonene samples produced in 2-butanol and NMP, named as $\mathrm{Sb}-\mathrm{BuOH}$ and Sb-NMP, respectively, as electrocatalysts for hydrogen evolution reaction (HER) under acidic $\mathrm{pH}$.

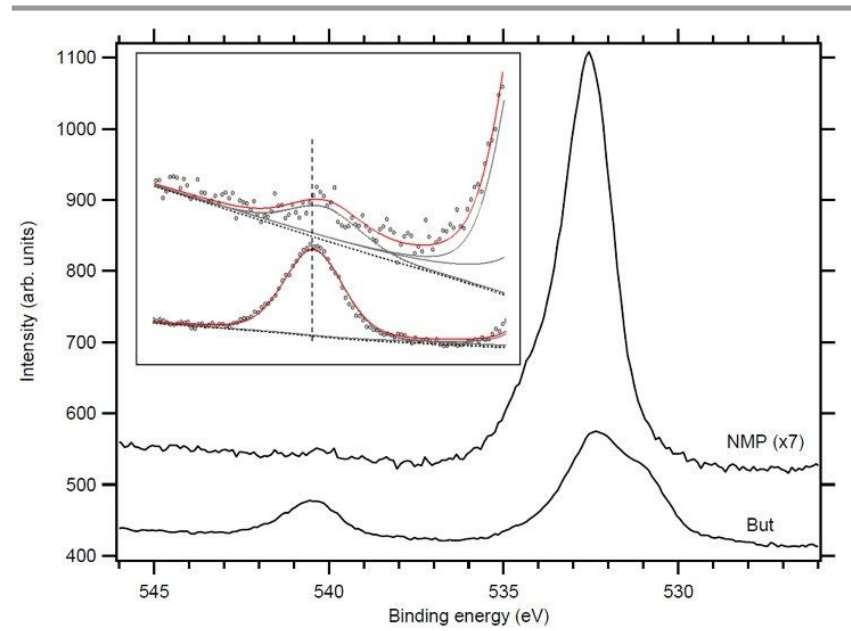

Fig6. XPS Sb 3d and O 1s region of the FL antimonene samples prepared using NMP and 2-butanol, spectra are offset and rescaled for sake of clarity. The inset shows a magnification of the $\mathrm{Sb} 3 d_{3 / 2}$ peak for both samples showing a shift to lower binding energies in the case of the sample prepared with NMP of $c a .0 .4 \mathrm{eV}$. Dots are experimental points, the red line is the results of fit, and solid lines are individual components.

To analyse the electrocatalytical performance of the samples, linear sweep voltammetry (LSV) measurements were carried out at $5 \mathrm{mV} \cdot \mathrm{s}^{-1}$ in a previously $\mathrm{N}_{2}$ purged $0.5 \mathrm{M} \mathrm{H}_{2} \mathrm{SO}_{4}$ aqueous solution (Fig.7a). In order to compare the electrochemical performance, two parameters were explored: the Tafel slope value and the overpotential required at a current density of $10 \mathrm{~mA} \cdot \mathrm{cm}^{-2}$ (Fig. $7 \mathrm{~b}-\mathrm{C}$ ). Sb-NMP possesses the lower Tafel slope and $\mathrm{Sb}-\mathrm{BuOH}$ owns the lower overpotential value ($0.70 \mathrm{~V}$ ), which is similar to that previously reported by Gusmão and co-workers for shear-exfoliated few-layer antimonene. ${ }^{44}$ The reduction in the onset potential could be connected to both the dimensions of the sheets and the oxidation degree. Thus, concerning the former, the $\mathrm{Sb}-\mathrm{BuOH}$ exhibits lower thickness and a superior anisotropic ratio leading to a larger number of edges as well as electroactive sites. However, the marked differences observed would not be explained onlyetaking into account this effect. DOI: 10.1039/C9TA06072C

To investigate the influence of oxidation, we have recorded cyclic voltammetry (CV) curves before and after the LSV measurements. Interestingly, CV curves recorded after LSV exhibit redox peaks located between 0.3 and $0.6 \mathrm{~V}$ vs RHE with a significant current difference (at least 4 times higher), showing a much more pronounced redox activity for the $\mathrm{Sb}-\mathrm{BuOH}$ sample (Fig.7d-e). In contrast, when measuring first the CVs, no peaks were observed for the Sb-NMP sample, but a small one at $0.1 \mathrm{~V}$ connected to $\mathrm{Sb}^{0}$ oxidation in the $\mathrm{Sb}-\mathrm{BuOH}$ sample, confirming the highest degree of exfoliation, which is related to a higher number of more reactive edge atoms (Fig. $7 \mathrm{f}-\mathrm{g}$ ). Moreover, the intensity of the observed peaks significantly decreases with cycling, indicative of the presence of irreversible processes and/or progressive mass loss. Concerning the peaks observed after the HER experiments, it could happen that the sample is reduced from its initial oxidized form and this oxidized specie cannot be reduced at superior potentials than $-0.3 \mathrm{~V}$ vs. $\mathrm{RHE}$. Once the reduction takes place, the reduced $\mathrm{Sb}$ is able to suffer redox processes in this potential range.

To shed light at this point, we have recorded CVs fixing the cathodic potential and varying the anodic limit (Fig.7h). A first oxidation peak is reached at $0.20-0.25 \mathrm{~V}$ vs. RHE but without its corresponding reduction peak. It could be related to the oxidation of $\mathrm{Sb}$ to $\mathrm{Sb}^{3+}$. The absence of a reduction peak could be explained by the formation of $\mathrm{SbO}^{+}$that is dissociated in the solution and/or the hydrogen oxidation, explaining the decrease in intensity observed in the CVs. From $0.30 \mathrm{~V}$ vs. RHE, a second oxidation peak (or combination of several ones) is observable with a reduction peak located between $-0.30-0.00$ $\mathrm{V}$ vs. RHE. This reduction does not appear until the oxidation reaction shows up revealing the connection between both peaks. The involved reaction could be the oxidation of $\mathrm{Sb}$ and the progressive reduction of $\mathrm{Sb}_{2} \mathrm{O}_{3}$ and/or other antimony oxides with higher oxidation degrees (i.e. $\mathrm{Sb}_{2} \mathrm{O}_{4}$ and $\mathrm{Sb}_{2} \mathrm{O}_{5}$ ). In order to evaluate the reversibility of the redox processes, the charge was calculated by integrating the oxidation and the reduction peaks. Comparing the two processes, one can conclude the existence of irreversible oxidation with a coefficient $\mathrm{Q}_{\mathrm{ox}} / \mathrm{Q}_{\text {red }}$ value of $1.5-2$ (†Fig.S8). Even if the oxidation charge considers the two reactions, the peak at 0.25 $\mathrm{V}$ vs. RHE has a charge of $c a .0 .04 \mathrm{mC}$, inferior to the ox/red difference, therefore indicative of the irreversible formation of antimonene oxides. Another issue to remark is the improvement of the HER upon the anodic limit increase. Then, it is possible that irreversible oxidation and then, the formation of $\mathrm{Sb}_{2} \mathrm{O}_{3}$ lead to higher electrocatalytic performance, being responsible for the better electrocatalytic behavior exhibited by the 2-butanol sample, highlighting the importance of controlling the oxidation degree in antimonene when exploring energy storage and conversion applications.

Furthermore, CVs were recorded fixing the anodic potential and varying the cathodic limit (Fig.7i). Before reducing, only an oxidation process can be observed. Indeed, another oxidation reaction appears when reduction potentials have been reached proving again these peaks are related. As described above, the 
sample could be in the form of a mixture of antimony oxides $\left(\mathrm{Sb}^{3+}\right.$ and $\left.\mathrm{Sb}^{5+}\right)$ being partially reduced to $\mathrm{Sb}^{0}$.

The initial oxidizing peak present in the pristine 2-butanol sample should be connected to the oxidation of $\mathrm{Sb}^{0}$, and it can be explained by the highest degree of exfoliation. In addition, similar to the previous case, irreversible oxidation can be noticed. This is very interesting, as indicates that the oxidation of antimonene may result in the formation of novel surface antimonene oxides with specific structural and physical properties.

Summarizing, Sb-BuOH presents higher HER performance than Sb-NMP likely due to its better exfoliation and anisotropic ratio giving a large number of edges and thus electroactive sites. Moreover, the HER performance seems to be related with the irreversible formation of superficial antimony oxides, which is much more pronounced in the case of $\mathrm{Sb}-\mathrm{BuOH}$ sample than in the Sb-NMP one, in good accordance with the XPS results.

\section{Conclusions}

We have reported on a systematic study that optimize several parameters of the exfoliation process of antimony bulk crystals with the aim to produce stable FL antimonene suspensions with high nanonlayers concentration as well as high DA ratio.

The results show that the pre-processing of the material affects both concentration and morphology of the suspension of FL antimonene, being the optimal process that using "wetball milling" with 2-butanol.

We have also observed that the solvent plays a crucial role in the LPE process. From the 28 solvents preselected based on Hansen parameters as well as other experimental observations, the highest concentration value so far reported was obtained using a mixture of $\mathrm{NMP} / \mathrm{H}_{2} \mathrm{O}$ in a (4:1) ratio, $c a .0 .368 \mathrm{~g} / \mathrm{L}$ ( yield of $37 \mathrm{wt} \%)$, while largest DA value was obtained using 2-butanol (ca. 27.6). Nonetheless both solvents seem to be suitable for most of the potential applications. Characterization of the nanolayers produced with these solvents show that in: i) 2 butanol $\langle\mathrm{L}\rangle=326.38 \mathrm{~nm},\langle\mathrm{H}\rangle=11.81 \mathrm{~nm}$ with $\sim 50 \%$ of the nanolayer with heights between $2-8 \mathrm{~nm}$, and lateral dimensions between $40-300 \mathrm{~nm}$, being the nanolayers with smallest height those of $c a .3 .6 \mathrm{~nm}$ and lateral dimensions of $c a .98 \mathrm{~nm}$; and ii) $\mathrm{NMP} /$ water $(4: 1)<\mathrm{L}>=292.51 \mathrm{~nm},\langle\mathrm{H}\rangle=15.79 \mathrm{~nm}$ with $50 \%$ of the nanolayer with heights between $2-10 \mathrm{~nm}$, and lateral dimensions between $60-250 \mathrm{~nm}$, being the nanolayers with smallest height those of $c a .4 .3 \mathrm{~nm}$ and lateral dimensions of $c a$. $72 \mathrm{~nm}$.

Finally, we have noticed that using a sonication tip compared to bath sonication yields better results, whilst employing an ultrasonic wave amplitude of $100 \%$ the concentration and the DA of the samples also increases. To conclude, HER activity of both Sb-BuOH and Sb-NMP has been characterized to illustrate the usefulness of this optimized LPE process, where $\mathrm{Sb}-\mathrm{BuOH}$ presents higher performance likely due to its better exfoliation and anisotropic ratio giving a large number of edges and electroactive sites. Interestingly, the HER performance seems to be also related with the irreversible formation of superficial antimony oxides, being pronounced in the case of Sb-BuOH.

DOI: 10.1039/C9TA06072C

This work provides an important insight into the factors influencing the LPE of antimonene, its structural characterization and electrochemical properties. Furthermore, these results serve as a guideline for the large-scale production of few-layers antimonene of great interest in catalysis, biomedicine, or energy storage and conversion.

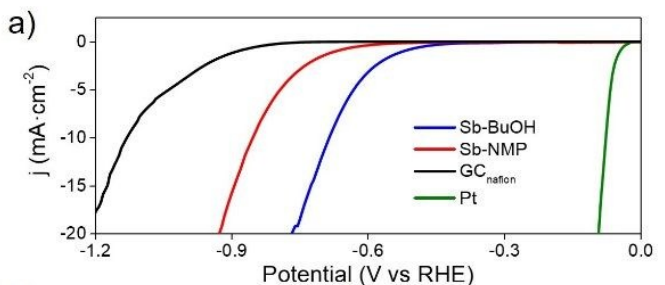

b)

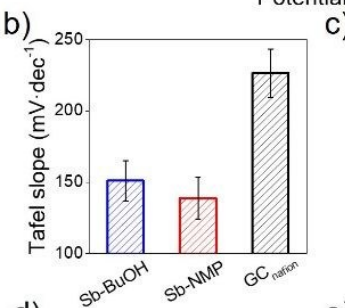

c) $\widehat{\underline{\omega}}$

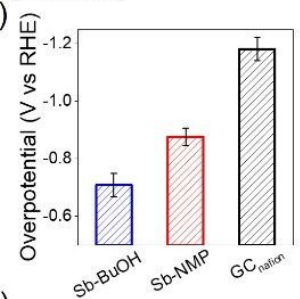

d)

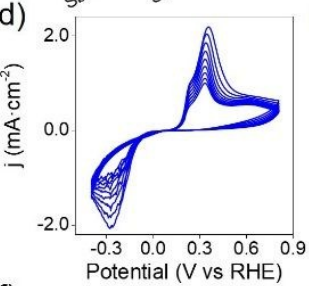

e)
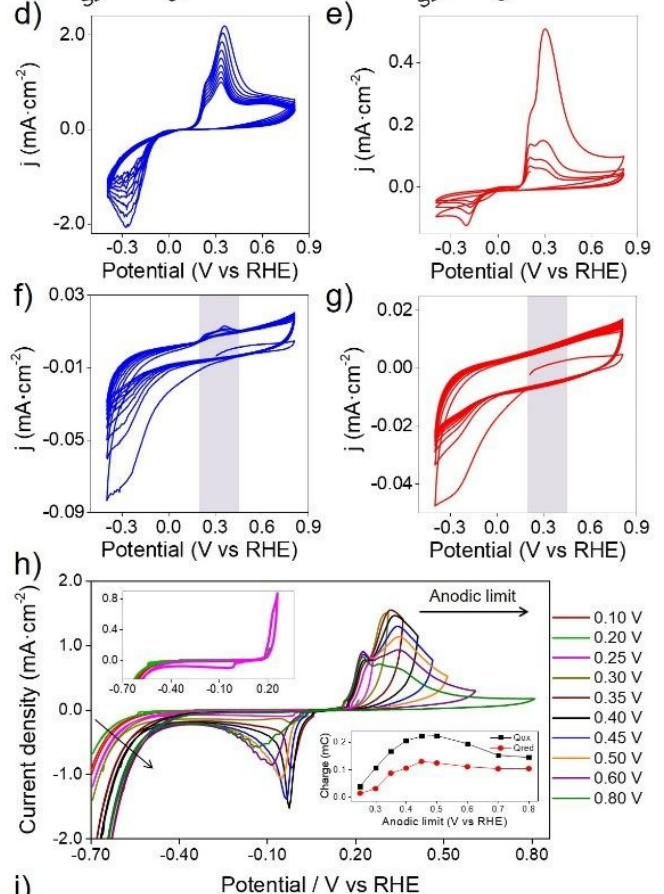

i)

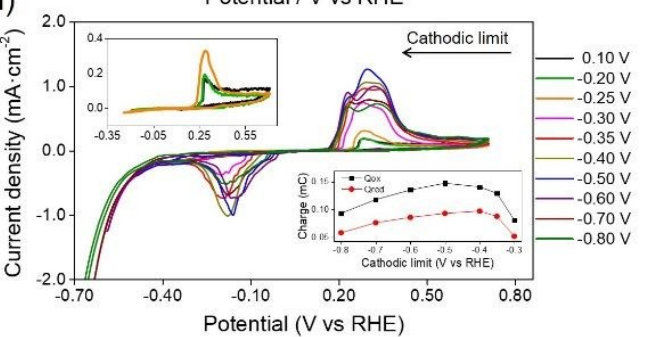

Fig 7. a) LSV curves of the different FL antimonene samples, nafion modified glassy carbon blank and platinum blank. b) Tafel slopes values and c) Overpotentials required for $10 \mathrm{~mA} \cdot \mathrm{cm}^{-2}$ calculated from LSV data. CVs recorded after the LSV measurements of the $\mathrm{Sb}-\mathrm{BuOH}$ (d) and Sb-NMP (e). CVs recorded before the LSV measurements of the Sb$\mathrm{BuOH}(\mathrm{f})$ and Sb-NMP samples (g). h) Voltammograms obtained between $-0.9 \mathrm{~V}$ and 
different anodic potential limits measured using $50 \mathrm{mV} \cdot \mathrm{s}^{-1}$. i) Voltammograms obtained between $0.5 \mathrm{~V}$ and different cathodic potential limits measured using $50 \mathrm{mV} \cdot \mathrm{s}^{-1}$. The insets show the calculated charges obtained when a positive current is passed $\left(Q_{o x}\right)$ or negative current is passed $\left(Q_{\text {red }}\right)$.

\section{Conflicts of interest}

There are no conflicts to declare.

\section{Acknowledgements}

The work has been supported by the European Union (ERC2018-StG 804110-2D-PnictoChem to G.A.) and the Spanish MICINN (MAT2016-77608-C3-1-P and PCI2018-093081) and MINECO (Structures of Excellence María de Maeztu MDM-20150538, FIS2017-82415-R). G.A. acknowledges support by the Generalitat Valenciana (CIDEGENT/2018/001) and the Deutsche Forschungsgemeinschaft (DFG; FLAG-ERA AB694/2-1). R.S-G. acknowledges the Spanish MINECO for a FPU fellowship. J.J.P. and W.S.P. acknowledge financial support from Spanish MINECO through Grant FIS2016-80434-P, the Fundación Ramón Areces, and the European Union Seventh Framework Programme under FLAG-ERA agreement No. 604391. W.S.P. was funded by the CNPq Fellowship programme (Pósdoutorado júnior) under grant 405107/2017-0 and acknowledges the computer resources and assistance provided by the Centro de Computación Científica of the Universidad Autónoma de Madrid and the RES.

\section{References}

1 K. S. Novoselov, Science, 2004, 306, 666-669.

2 K. S. Novoselov, D. Jiang, F. Schedin, T. J. Booth, V. V Khotkevich, S. V Morozov and a K. Geim, Proc. Natl. Acad. Sci., 2005, 102, 10451-10453.

3 Q. H. Wang, K. Kalantar-Zadeh, A. Kis, J. N. Coleman and M. S. Strano, Nat. Nanotechnol., 2012, 7, 699-712.

4 R. Mas-Ballesté, C. Gómez-Navarro, J. Gómez-Herrero and F. Zamora, Nanoscale, 2011, 3, 20-30.

5 A. Harvey, J. B. Boland, I. Godwin, A. G. Kelly, B. M. Szydłowska, G. Murtaza, A. Thomas, D. J. Lewis, P. O’Brien and J. N. Coleman, 2D Mater., 2017, 4, 025054.

6 M. Zeng, Y. Xiao, J. Liu, K. Yang and L. Fu, Chem. Rev., 2018, 118, 6236-6296.

7 X. Cai, Y. Luo, B. Liu and H. M. Cheng, Chem. Soc. Rev., 2018, 47, 6224-6266.

8 A. Castellanos-Gomez, L. Vicarelli, E. Prada, J. O. Island, K. L. Narasimha-Acharya, S. I. Blanter, D. J. Groenendijk, M. Buscema, G. a. Steele, J. V. Alvarez, H. W. Zandbergen, J. J. Palacios and H. S. J. van der Zant, 2 D Mater., 2014, 1, 025001.

9 S. Zhang, X. Zhang, L. Lei, X.-F. Yu, J. Chen, C. Ma, F. Wu, Q. Zhao and B. Xing, Angew. Chem., Int. Ed., 2019, 58, 467-471.

10 Q. Zhou, Q. Chen, Y. Tong and J. Wang, Angew. Chem., Int. Ed., 2016, 55, 11437-11441.

11 A. Favron, E. Gaufrès, F. Fossard, A. L. Phaneuf-Laheureux, N. Y. W. Tang, P. L. Lévesque, A. Loiseau, R. Leonelli, S.
Francoeur and R. Martel, Nat. Mater., 2015, 14 826-832 ine

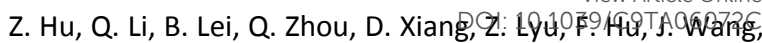
Y. Ren, R. Guo, E. Goki, L. Wang, C. Han, J. Wang and W. Chen, Angew. Chem., Int. Ed., 2017, 56, 9131-9135.

G. Abellán, S. Wild, V. Lloret, N. Scheuschner, R. Gillen, U. Mundloch, J. Maultzsch, M. Varela, F. Hauke and A. Hirsch, J. Am. Chem. Soc., 2017, 139, 10432-10440.

S. Zhang, S. Guo, Z. Chen, Y. Wang, H. Gao, J. GómezHerrero, P. Ares, F. Zamora, Z. Zhu and H. Zeng, Chem. Soc. Rev., , DOI:10.1039/C7CS00125H.

P. Ares, J. J. Palacios, G. Abellán, J. Gómez-Herrero and F. Zamora, Adv. Mater., 2018, 30, 1703771. S. Zhang, Z. Yan, Y. Li, Z. Chen and H. Zeng, Angew. Chem., Int. Ed., 2015, 54, 3112-3115.

P. Ares, F. Aguilar-Galindo, D. Rodríguez-San-Miguel, D. A. Aldave, S. Díaz-Tendero, M. Alcamí, F. Martín, J. GómezHerrero and F. Zamora, Adv. Mater., 2016, 28, 6332-6336. C. Gibaja, D. Rodriguez-San-Miguel, P. Ares, J. GómezHerrero, M. Varela, R. Gillen, J. Maultzsch, F. Hauke, A. Hirsch, G. Abellán and F. Zamora, Angew. Chem., Int. Ed., 2016, 55, 14345-14349.

19 F. Li, M. Xue, J. Li, X. Ma, L. Chen, X. Zhang, D. R. MacFarlane and J. Zhang, Angew. Chem., Int. Ed., 2017, 56, 1471814722.

20 J. Gu, Z. Du, C. Zhang, J. Ma, B. Li and S. Yang, Adv. Energy Mater., 2017, 7, 1-8.

21 W. Tian, S. Zhang, C. Huo, D. Zhu, Q. Li, L. Wang, X. Ren, L. Xie, S. Guo, P. K. Chu, H. Zeng and K. Huo, ACS Nano, 2018, 12, 1887-1893.

G. Abellán, P. Ares, S. Wild, E. Nuin, C. Neiss, D. R.-S. Miguel, P. Segovia, C. Gibaja, E. G. Michel, A. Görling, F. Hauke, J. Gómez-Herrero, A. Hirsch and F. Zamora, Angew. Chem., Int. Ed., 2017, 56, 14389-14394.

W. Tao, X. Ji, X. Zhu, L. Li, J. Wang, Y. Zhang, P. E. Saw, W. Li, N. Kong, M. A. Islam, T. Gan, X. Zeng, H. Zhang, M. Mahmoudi, G. J. Tearney and O. C. Farokhzad, Adv. Mater., 2018, 30, 1802061.

24 E. Martínez-Periñán, M. P. Down, C. Gibaja, E. Lorenzo, F. Zamora and C. E. Banks, Adv. Energy Mater., 2018, 8, 1702606.

V. Lloret, M. Á. Rivero-Crespo, J. A. Vidal-Moya, S. Wild, A. Doménech-Carbó, B. S. J. Heller, S. Shin, H. Steinrück, F. Maier, F. Hauke, M. Varela, A. Hirsch, A. Leyva-Pérez and G. Abellán, Nat. Commun., 2019, 10, 509.

V. Nicolosi, M. Chhowalla, M. G. Kanatzidis, M. S. Strano and J. N. Coleman, Science, 2013, 340, 1226419-1226419.

K. R. Paton, E. Varrla, C. Backes, R. J. Smith, U. Khan, A. O'Neill, C. Boland, M. Lotya, O. M. Istrate, P. King, T. Higgins, S. Barwich, P. May, P. Puczkarski, I. Ahmed, M. Moebius, H. Pettersson, E. Long, J. Coelho, S. E. O'Brien, E. K. McGuire, B. M. Sanchez, G. S. Duesberg, N. McEvoy, T. J. Pennycook, C. Downing, A. Crossley, V. Nicolosi and J. N. Coleman, Nat. Mater., 2014, 13, 624-30.

A. Puthirath Balan, S. Radhakrishnan, C. F. Woellner, S. K. Sinha, L. Deng, C. D. L. Reyes, B. M. Rao, M. Paulose, R. Neupane, A. Apte, V. Kochat, R. Vajtai, A. R. Harutyunyan, C. 
W. Chu, G. Costin, D. S. Galvao, A. A. Martí, P. A. van Aken, O. K. Varghese, C. S. Tiwary, A. Malie Madom Ramaswamy Iyer and P. M. Ajayan, Nat. Nanotechnol., 2018, 13, 1-8.

29 J. N. Coleman, Adv. Funct. Mater., 2009, 19, 3680-3695.

30 Y. Hernandez, V. Nicolosi, M. Lotya, F. M. Blighe, Z. Sun, S. De, I. T. McGovern, B. Holland, M. Byrne, Y. K. Gun'Ko, J. J. Boland, P. Niraj, G. Duesberg, S. Krishnamurthy, R. Goodhue, J. Hutchison, V. Scardaci, A. C. Ferrari and J. N. Coleman, Nat. Nanotechnol., 2008, 3, 563-568.

31 I. Horcas, R. Fernández, J. M. Gómez-Rodríguez, J. Colchero, J. Gómez-Herrero and A. M. Baro, Rev. Sci. Instrum., 2007, 78, 013705.

32 P. Nemes-Incze, Z. Osváth, K. Kamarás and L. P. Biró, Carbon N. Y., 2008, 46, 1435-1442.

33 M. Schmid, H. P. Steinrück and J. M. Gottfried, Surf. Interface Anal., 2014, 46, 505-511.

34 X. Sun, Z. Song, S. Liu, Y. Wang, Y. Li, W. Wang and J. Lu, ACS Appl. Mater. Interfaces, 2018, 10, 22363-22371.

35 H. Tao, Y. Zhang, Y. Gao, Z. Sun, C. Yan and J. Texter, Phys. Chem. Chem. Phys., 2017, 19, 921-960.

36 J. N. Coleman, M. Lotya, A. O'Neill, S. D. Bergin, P. J. King, U. Khan, K. Young, A. Gaucher, S. De, R. J. Smith, I. V. Shvets, S. K. Arora, G. Stanton, H. Y. Kim, K. Lee, G. T. Kim, G. S. Duesberg, T. Hallam, J. J. Boland, J. J. Wang, J. F. Donegan, J. C. Grunlan, G. Moriarty, A. Shmeliov, R. J. Nicholls, J. M. Perkins, E. M. Grieveson, K. Theuwissen, D. W. McComb, P. D. Nellist and V. Nicolosi, Science, 2011, 331, 568-571.

C. Backes, T. M. Higgins, A. Kelly, C. Boland, A. Harvey, D. Hanlon and J. N. Coleman, Chem. Mater., 2017, 29, 243255.

38 J. A. Carrasco, A. Harvey, D. Hanlon, V. Lloret, D. McAteer, R. Sanchis-Gual, A. Hirsch, F. Hauke, G. Abellán, J. N. Coleman and E. Coronado, Chem. Commun., 2019, 55, 3315-3318.

39 X. Wang, J. He, B. Zhou, Y. Zhang, J. Wu, R. Hu, L. Liu, J. Song and J. Qu, Angew. Chem., Int. Ed., 2018, 57, 8668-8673.

40 Y. Hernandez, M. Lotya, D. Rickard, S. D. Bergin and J. N. Coleman, Langmuir, 2010, 26, 3208-3213.

41 M. Fortin-Deschênes, O. Waller, T. O. Menteş, A. Locatelli, S. Mukherjee, F. Genuzio, P. L. Levesque, A. Hébert, R. Martel and O. Moutanabbir, Nano Lett., 2017, 17, 4970-4975.

42 D. Hanlon, C. Backes, E. Doherty, C. S. Cucinotta, N. C. Berner, C. Boland, K. Lee, A. Harvey, P. Lynch, Z. Gholamvand, S. Zhang, K. Wang, G. Moynihan, A. Pokle, Q. M. Ramasse, N. McEvoy, W. J. Blau, J. Wang, G. Abellan, F. Hauke, A. Hirsch, S. Sanvito, D. D. O’Regan, G. S. Duesberg, V. Nicolosi and J. N. Coleman, Nat. Commun., 2015, 6, 8563.

43 J. Kang, J. D. Wood, S. A. Wells, J.-H. Lee, X. Liu, K.-S. Chen and M. C. Hersam, ACS Nano, 2015, 9, 3596-3604.

44 R. Gusmão, Z. Sofer, D. Bouša and M. Pumera, Angew. Chem., Int. Ed., 2017, 56, 14417-14422. 


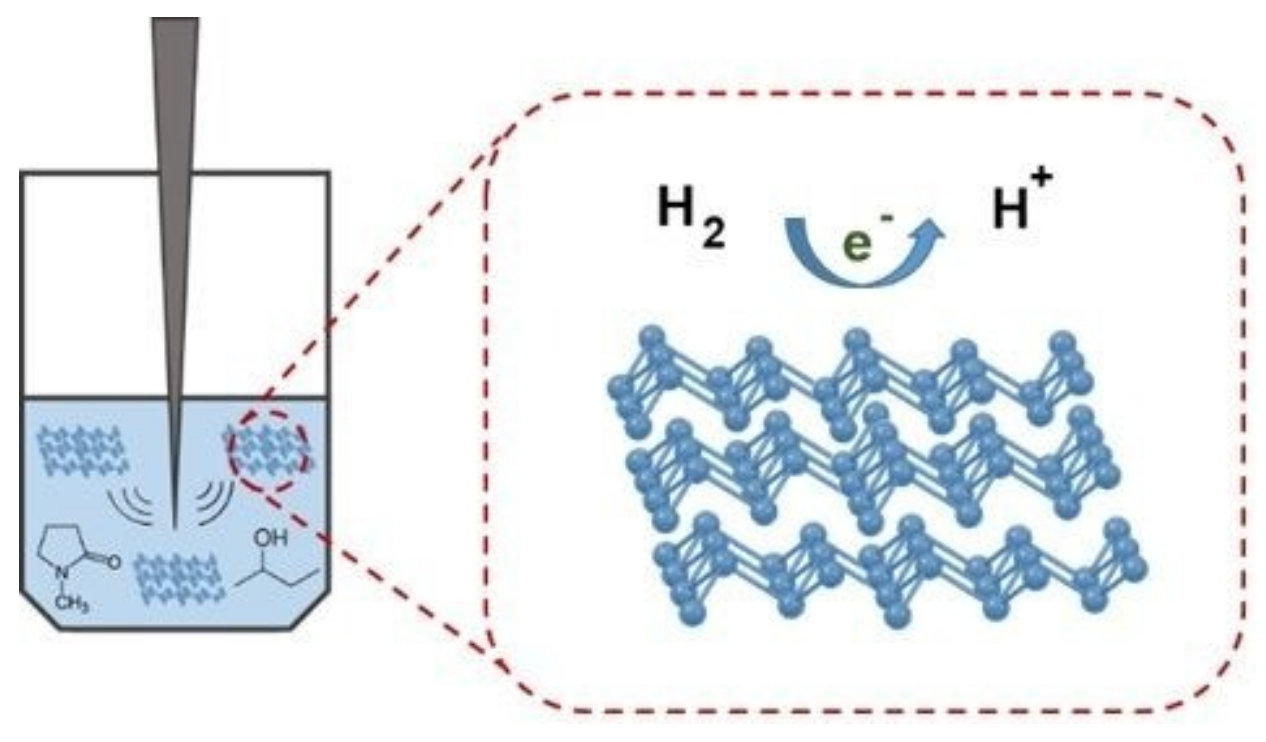

$125 \times 71 \mathrm{~mm}(96 \times 96 \mathrm{DPI})$ 